\title{
A combined XRF/Monte Carlo simulation study of multilayered Peruvian metal artifacts from the tomb of the Priestess of Chornancap
}

\author{
Antonio Brunetti ${ }^{1} \cdot$ Julio Fabian $^{2} \cdot$ Carlos Wester La Torre $^{3} \cdot$ Nick Schiavon $^{4}$
}

Received: 1 December 2015/ Accepted: 24 April 2016

(C) Springer-Verlag Berlin Heidelberg 2016

\begin{abstract}
An innovative methodological approach based on XRF measurements using a polychromatic X-ray beam combined with simulation tests based on an ultra-fast custom-made Monte Carlo code has been used to characterize the bulk chemical composition of restored (i.e., cleaned) and unrestored multilayered Peruvian metallic artifacts belonging to the twelfth- and thirteenth-century AD funerary complex of Chornancap-Chotuna in northern Peru. The multilayered structure was represented by a metal substrate covered by surface corrosion patinas and/or a layer from past protective treatments. The aim of the study was to assess whether this new approach could be used to overcome some of the limitations highlighted in previous research performed using monochromatic X-ray beam on patina-free and protective treatment-free metal artifacts in obtaining reliable data both on the composition on the bulk metals and on surface layers thickness. Results from the analytical campaign have led to a reformulation of previous hypotheses about the structure and composition of the metal used to create the Peruvian artifacts under investigation.
\end{abstract}

Nick Schiavon

schiavon@uevora.pt

Polcoming Department, University of Sassari, Sassari, Italy

2 Facultad de Ciencias Físicas, Universidad Nacional Mayor de San Marcos, Lima, Peru

3 Museo Arqueológico Nacional Brüning, Lambayeque, Peru

4 Hercules Laboratory, University of Évora, Évora, Portugal

\section{Introduction}

In late 2011, excavations directed by the archeologist Carlos Wester La Torre, director of the Brüning National Archaeological Museum of the Ministry of Culture of Peru, discovered a new funerary complex in the ChornancapChotuna archeological site situated in the region of Lambayeque, on the north coast of Peru. Anthropological analysis suggested the human remains as belonging to a woman between 25 and 30 years old who lived in the twelfth and thirteenth centuries $\mathrm{AD}$, corresponding to the last period of the Lambayeque culture. The Chornancap Priestess, as she was named by the discoverers, was buried together with a vast array of metal artifacts including gold and copper jewelry (Fig. 1). A gold scepter was also found confirming the high rank of the person buried at the site. All the metal objects belonging to the trousseau of the priestess displayed the highest quality in terms of workmanship and beauty. The tomb had been submerged for about eight centuries below groundwater level, making it the first known occurrence in Latin America of a water-filled tomb. In recent years, other tombs of high-ranked personalities have been discovered in the area adding importance to the whole site and increasing our knowledge of the Lambayeque culture.

In this paper, an X-ray fluorescence (XRF) study of selected metal objects found in the burial is reported and discussed. Here we use XRF measurements together with simulations performed with a custom fast Monte Carlo code. This approach allows a more accurate analysis of the sample in any experimental condition, as in the model, an oxide layer as well as a protective layer can be easily introduced. A better description of the method used will be given in the next section.

$\mathrm{XRF}$ is a non destructive analytical technique well known in Cultural Heritage as well as in many other 
research areas [1-11]. Performing quantitative XRF analyses on archeological and Cultural Heritage metallic artifacts is, however, a particularly challenging task due to their often complex multilayered internal structures where "layers" may include a substrate made up of different metals (i.e., gold on silver, gold on copper) intentionally juxtaposed by the object makers, surface patinas caused by environmental corrosion agents (rich in Ag sulfides, oxides and/or chlorides compounds) and/or protective treatments applied in past conservation interventions [12-14]. In cases where both the separation between two or more adjacent layers is not well defined and/or when the layers are very thin, meaningful results on the bulk metal composition are even more difficult to obtain. In order to estimate the layer thickness and composition in multilayered archeological and Cultural Heritage metal materials, a standard method has been proposed in recent years by Cesareo et al. [15]: This methodological approach was based on estimating changes in the theoretical ratios of selected fluorescence line intensities due to the attenuation of the layers crossed by the radiation. However, this method is strongly dependent on the XRF setup used (monochromatic X-ray beam) and on the level of monochromatization of the X-ray beam obtained by placing a filter in the output of the X-ray tube which in turn affects the quality of the results. Moreover, in these studies, the metal objects were analyzed after cleaning and removal of any outer corrosion patinas (often containing corrosion products in the form of metal chlorides, sulfides and oxides) and/or of protective layers present. The methodological approach adopted in the current study involving Monte Carlo simulation tests does not require any constraint on the setup or on the XRF excitation spectrum. Moreover, it can and has been applied also to a unique set of metal artifacts before and without the need for cumbersome and potentially damaging cleaning procedures [12].

\section{Methods}

The shape of the spectrum exciting the sample depends on the kind of interactions occurred. One of the possible results of the photoelectric interaction is the production of a fluorescence photon. These photons will be represented by a peak centered around the characteristic energy on the detected spectrum. The other types of interaction will essentially produce a background superimposed to the peaks. Both fluorescence peaks and background are important for sample characterization. In the case of peaks, the area under the peak is roughly related to the percentage of the chemical contents inside the sample, while the scattering contribution can be also attributed to that part of the sample content that does not produce peaks, the so- called obscure matrix of the sample. In the case of the metallic object discussed here, the scattering is mainly due to the protective film and the oxidation patina and so can be used, for example, to determine the thickness of such layers. For this reason and unlike other approaches reported in the literature, we have used a polychromatic beam [5, 7]. Our approach is based on the use of a Monte Carlo (MC) simulation following a probabilistic simulation of the X-ray interaction with matter [16-23]. Usually, several millions of photons are simulated in order to be able to reproduce a good simulation of real experiments. These codes are able to simulate a variety of X-ray experimental setups, but this lack of specialization has the drawback that, in order to achieve an adequate simulation of an XRF analysis, a considerable amount of simulation time (in the order of several hours or days) is usually required. To overcome this problem, several specialized MC codes have been developed. They are able to simulate a reduced set of X-ray experiments, say for energy less than $100 \mathrm{keV}$. Nevertheless, this limitation does not introduce a true restraint on the XRF experiments that can be performed, because these are usually performed at energies around 40-50 keV, at least for Cultural Heritage applications. The great advantage is a dramatic increase in the speed of the simulation run with an XRF analysis adequately simulated in just a couple of minutes. This time is comparable to the experimental acquisition time and so, in such a sense, the simulation can be regarded as performed in real time. There are, to the best of our knowledge, two of such fast MC codes [20-23]. The first has been developed just for XRF experiments, while the second one can be also used to simulate radiographic, CT and phase contrast simulations. Both codes are based on the Xraylib database [24, 25]. Here we use the second one called XRMC [20, 21]. Before running the simulation, any $\mathrm{MC}$ code requires a detailed description of both sample's composition and structure. From the point of view of the authors, one of the most critical parameters in this respect is the X-ray spectrum emitted by the source. Here we use the real spectrum emitted from the source, corrected for the air attenuation as well as for the tube and detector windows attenuations. Its correctness has been tested on several well-known artifacts. The estimate of the quality of the simulation is evaluated by comparing the simulated and measured spectra. When the simulated spectrum matches the experimental one almost exactly, the sample will be determined in both composition and structures. The first part of comparison is based just on visual observation of the spectra, which is then further tested by a Chi-squared test. The match must be as much accurate as possible, because there could be several sets of compositions and structures able to produce a spectrum similar to the experimental one and, in this sense, several different models must be tested: only the 
simulation of structure/composition producing the spectrum nearest to the experimental one will be considered as the correct one. The accuracy of the matching is particularly important in the case of multilayered structures where an element can be present in more than one layer such as, for example, in the presence of surface-corroded metals like the Peruvian metallic artifacts under investigation. $X R M C$ is also able to simulate arbitrarily rough surfaces that are often found in Cultural Heritage samples. This code has been used jointly with a portable X-ray instrumentation. The use of portable XRF systems is mandatory when the samples cannot be moved from their storage site. The portable X-ray instrumentation used here is formed by an SDD coupled with a DSP multichannel analyzer (1-2-3 system manufactured by Amptek) and an Ag anode X-ray tube (mini-X manufactured by Amptek). The detector is placed vertically in front of sample $\left(90^{\circ}\right)$, and the X-ray tube is placed at about $30^{\circ}$, both $2-3 \mathrm{~cm}$ from the sample surface, depending on the accessibility of the sample. The system is connected to a notebook computer where the Monte Carlo code is also stored. Each Monte Carlo simulation lasts 1-2 min, producing a simulation of quality comparable to the experimental spectrum where the acquisition takes 4-6 min.

\section{Results and discussion}

The following objects found in the Priestess sepulture were analyzed: two bimetallic vases, one of which underwent restoration, and three earrings. The analysis of the two vases is particularly important because they allowed to test the Monte Carlo capability to simulate multilayered structures even before restoration as is the case with the unrestored vase. Both vases are characterized by a series of gold and silver areas (Fig. 2). Besides the layer of oxides

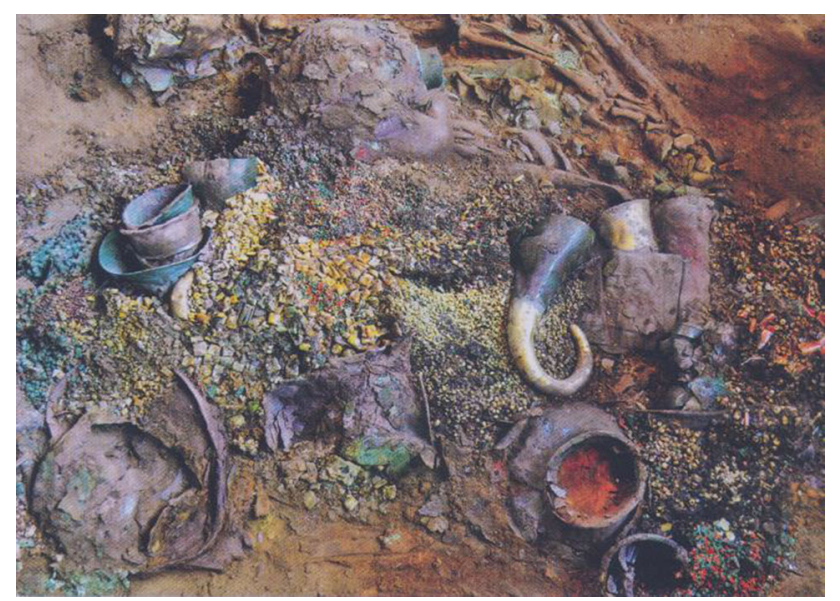

Fig. 1 Priestess of Chornancap sepulture covering parts of one of the vases (silver alloy zones), each zone, whether restored or not, can be considered as a multilayered one with each layer characterized by more than one chemical element. This typology is typical of Peruvian metallic artifacts, which often display a specific multilayered structure, i.e., that of a gilded metal and/or of the so-called Tumbaga gold artifacts. The latter consist of a particular type of multilayered objects which are formed by a gold layer at the surface superimposed to a copper-gold alloy (or, more rarely, silver-gold alloy) substrate. The superficial gold layer was probably obtained by chemically attacking the surface of the alloy (following a yet unknown methodology) and removing in the case of a gold and copper alloy, for example, the copper from the surface. The structure obtained in such a way appears to be made of pure gold, and it preserves its original aspect unaltered until today, while the gilded artifacts show evident signs of corrosion. Each vase has been examined in all its parts composing it and also at the interfaces between two adjacent zones in order to detect the presence of any different material used as a soldering, as suggested by the archeologist. However, some extraneous material, such as lead, was found.

In Fig. 3, the experimental setup and the spectrum acquired from the unrestored vase at the corroded silver surface are reported. The experimental spectrum appears to be composed essentially by copper (Fig. 3b). The MC fit is practically identical to the experimental one, except for the pile-up peaks around $15 \mathrm{keV}$ and the escape peak around $7 \mathrm{keV}$, which the MC code is not able to simulate in its present version. Several tests on different structure/composition models have been performed, including a gradientlike model based on a seven-layer structure. In the model, each layer is considered to be homogeneous. This could be considered as a limitation because the composition of metallic artifacts is often not homogenous, but it can be overcome by taking measurements at several points on the surface and, in this sense, the estimate obtained should be considered as representing the bulk composition of the

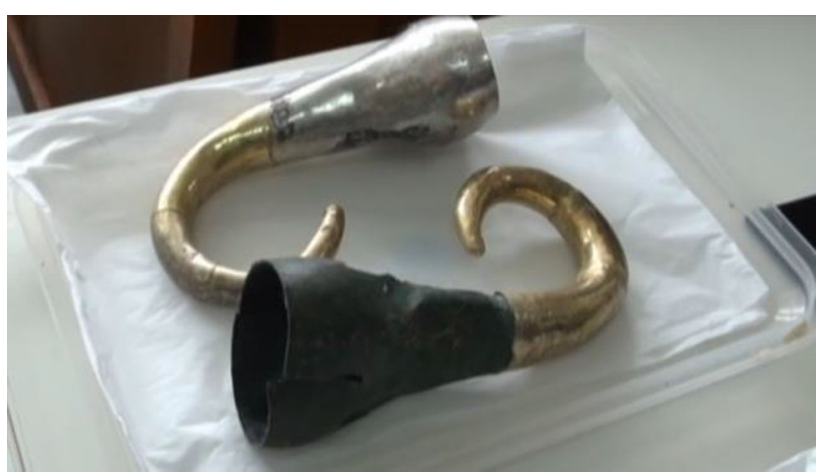

Fig. 2 Bimetallic vases 

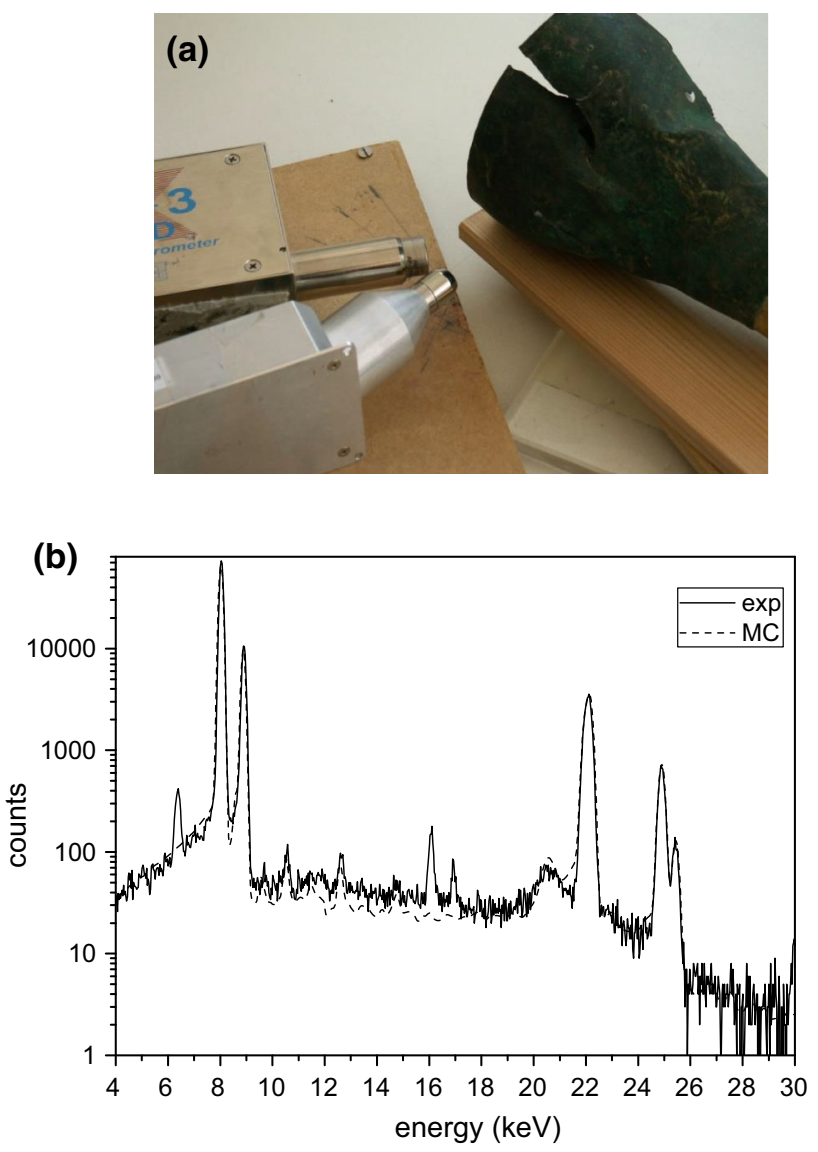

Fig. 3 Unrestored $\mathrm{Au}-\mathrm{Ag}$ vase. XRF measurements of the oxidized silver surface. a Setup; b experimental and simulated spectra

sample. For the spectrum reported in Fig. 3, the best Monte Carlo model is assuming the presence of a $20-\mu \mathrm{m}$-thick corrosion layer with $40 \%$ of $\mathrm{Cu}$ and $10 \%$ of $\mathrm{Ag}$, and a second layer formed by $95 \%$ of $\mathrm{Ag}, 3 \%$ of $\mathrm{Cu}, 1 \%$ of $\mathrm{Au}$, $0.5 \%$ of $\mathrm{Pb}, 0.2 \%$ of $\mathrm{Br}$ and $0.05 \%$ of $\mathrm{Fe}$, i.e., an almost pure Ag silver but with still some contribution from the corrosion patinas as evidenced by the presence of $\mathrm{Br}$ which has been reported as being often present in such patinas [26]. The high abundance of $\mathrm{Cu}$ at the surface can be explained by $\mathrm{Cu}$ migration from the inner layer.

The experimental setup and the spectra acquired at the silver surface of the restored vase are shown in Fig. $4 a, b$. Compared to the spectrum in Fig. 3b, where the corrosion layer was superimposed to the silver alloy, here the silver contribution is considerably stronger than the copper one. Moreover, the surface has been covered by a protective layer (Paraloid-like). In Fig. 4c, the MC simulation is shown as superimposed to the experimental spectrum. In this case, the multilayered model used is formed by a Paraloid layer superimposed to a silver alloy layer. The MC simulation estimates the thickness of the protective layer as being $10 \mu \mathrm{m}$ thick. The underlying layer is formed

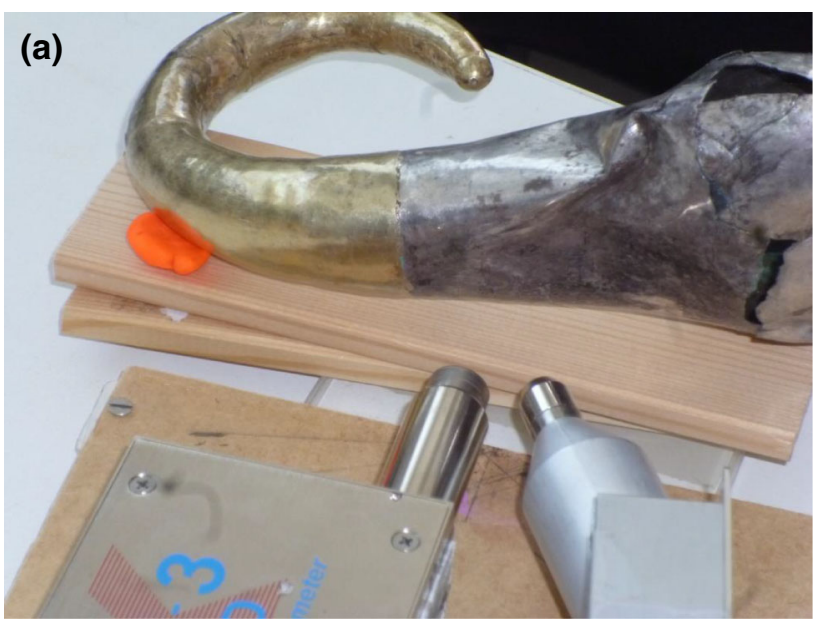

(b)

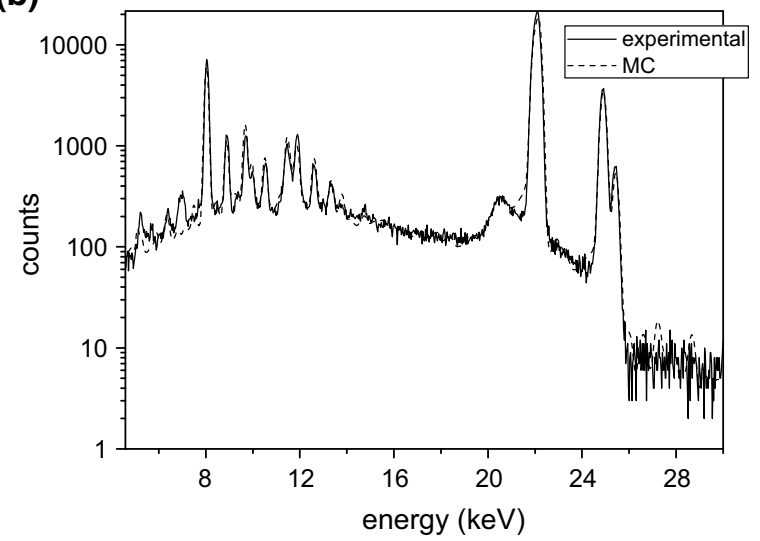

Fig. 4 Restored $\mathrm{Au}-\mathrm{Ag}$ vase. XRF measurements of the silver surface. a Setup; b experimental and simulated spectra

by $\mathrm{Ag}(93 \%), \mathrm{Cu}(5 \%), \mathrm{Au}(1 \%)$ and $\mathrm{Pb}(0.5 \%)$. This result is similar to the one obtained from the oxidized silver surface from the other vase. Thus, the Monte Carlo simulation proves itself as a valid auxiliary tool for the restorer. Of course, the estimate cannot be not as precise when the same material is present in more than one layer. In this case, the relative amounts of the same chemical element in the two layers can change without observable changes in the simulated spectrum, especially if it is in trace concentration in one of the layers. However, the error cannot be large due to the effect of the top-layer absorption on the underlying layer emission, especially at low energies. Let us explain this better. Let us suppose that a gold layer is superimposed to a copper layer and $\mathrm{X}$-ray photons from the $\mathrm{Cu}$ layer $\left(\mathrm{Cu}-\mathrm{K}_{\alpha}\right.$ and $\left.\mathrm{Cu}-\mathrm{K}_{\beta}\right)$ are emitted. These photons, in order to be detected, must travel across the gold layer to reach the detector. The rate of attenuation from a material is energy dependent and photons at lower energy $\left(\mathrm{Cu}-\mathrm{K}_{\alpha}\right)$ will be attenuated more than those at higher energy $(\mathrm{Cu}-$ $\mathrm{K}_{\beta}$ ). Thus, the intensity of each peak detected will be 
different from the one expected from a pure $\mathrm{Cu}$ object without any surface layer. This effect makes it possible to predict the thickness of the attenuating layer. Just to explain better this approach, two examples are shown in Fig. 5. The sample simulated is a multilayered one representing a real case: a Paraloid layer with $\mathrm{Ag}$ contamination, an almost pure gold layer and finally an $\mathrm{Ag}-\mathrm{Cu}-\mathrm{Au}$ alloy as a third layer. This simulation is compared to the one coming from a sample composed only by the third layer. The two K-line peaks of $\mathrm{Cu}$ undergo different attenuations with respect to the monolayer sample, with the $\mathrm{K}_{\alpha}$ more attenuated with respect to the $K_{\beta}$. This is due to the position of $\mathrm{Cu}$ in the multilayered structure. In this case, the $\mathrm{Cu}$ is present only in the second and in the third layer and so its fluorescence emission is attenuated by the first and second layers. In Fig. 5b, the effect on the attenuation is shown on the Ag L- and K-lines of the simulated spectrum. In this case, two effects can be observed: the lack of simulated Ag L-lines around $3 \mathrm{keV}$ and the different attenuation of the

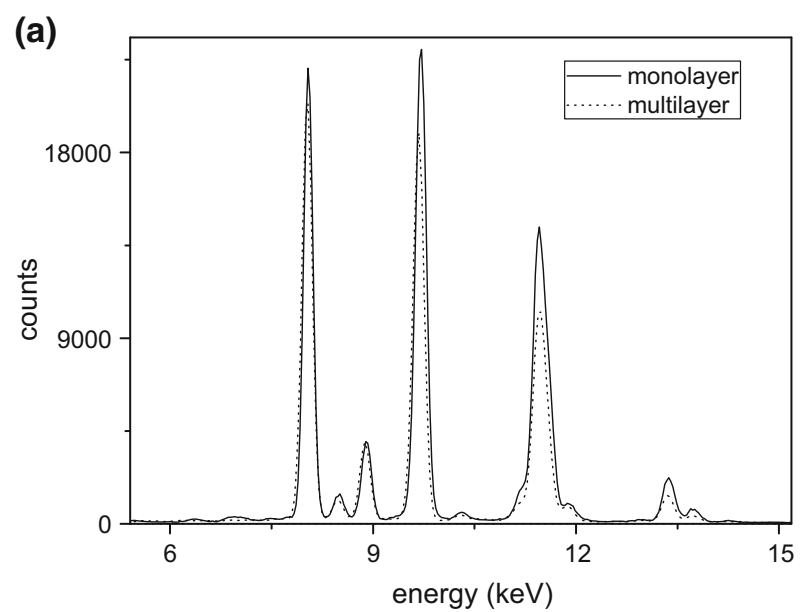

(b)

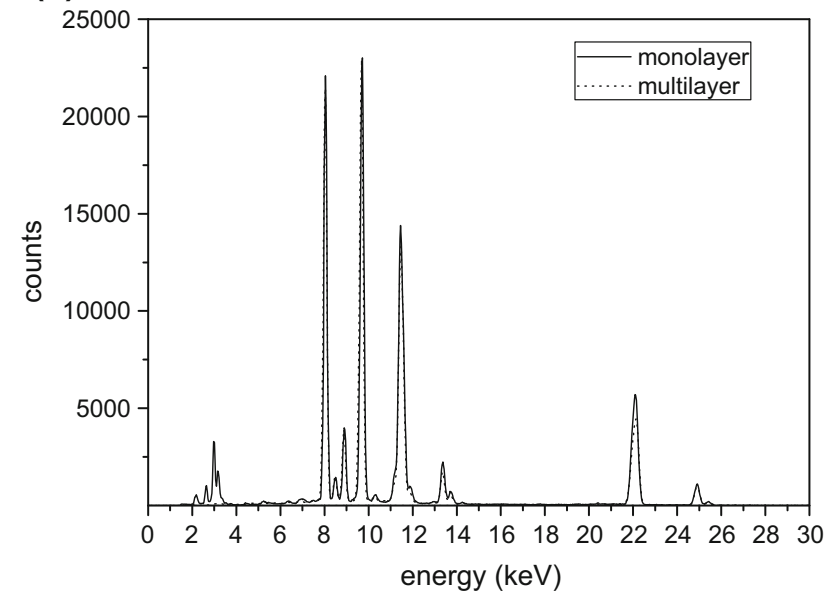

Fig. 5 Effect of the layer attenuation on a multilayer structure vs a monolayer. a Cu-K peaks attenuation by Au layer; b Ag-K and $\mathrm{Ag}-\mathrm{L}$ peaks attenuation by Au layer
Ag K-lines. The first effect reveals that, in the real sample, the silver must be present just in the first layer because of the very low energies of the Ag L-lines that cannot be detected if the silver is present only in the second and/or the third layers, due to the attenuation of the first layer. Of course, this does not hold true for any kind of sample. In fact, if the first layer is very thin and/or is made up of light elements, the Ag L-lines from the second layer could be detected, even if the effect of the attenuation of the first layer would be always noticeable. The second effect, being the $\mathrm{Ag} \mathrm{K}$-lines more penetrating than the $\mathrm{Ag}$ L-lines, indicates the effect of the attenuation of the first and/or the second layer if $\mathrm{Ag}$ is present at least in the third layer. Let us come back to the sample depicted in Fig. 4. The $\mathrm{Cu}$ in the oxide layer will be attenuated in a different way than the one present in the Ag layer. Thus, the $\mathrm{Cu}$ concentration in each layer of the model must be modulated according to the relative $\mathrm{Cu}$ peak amplitudes. In any case, it is always mandatory to obtain statistically relevant data, i.e., intense, well-defined peaks.
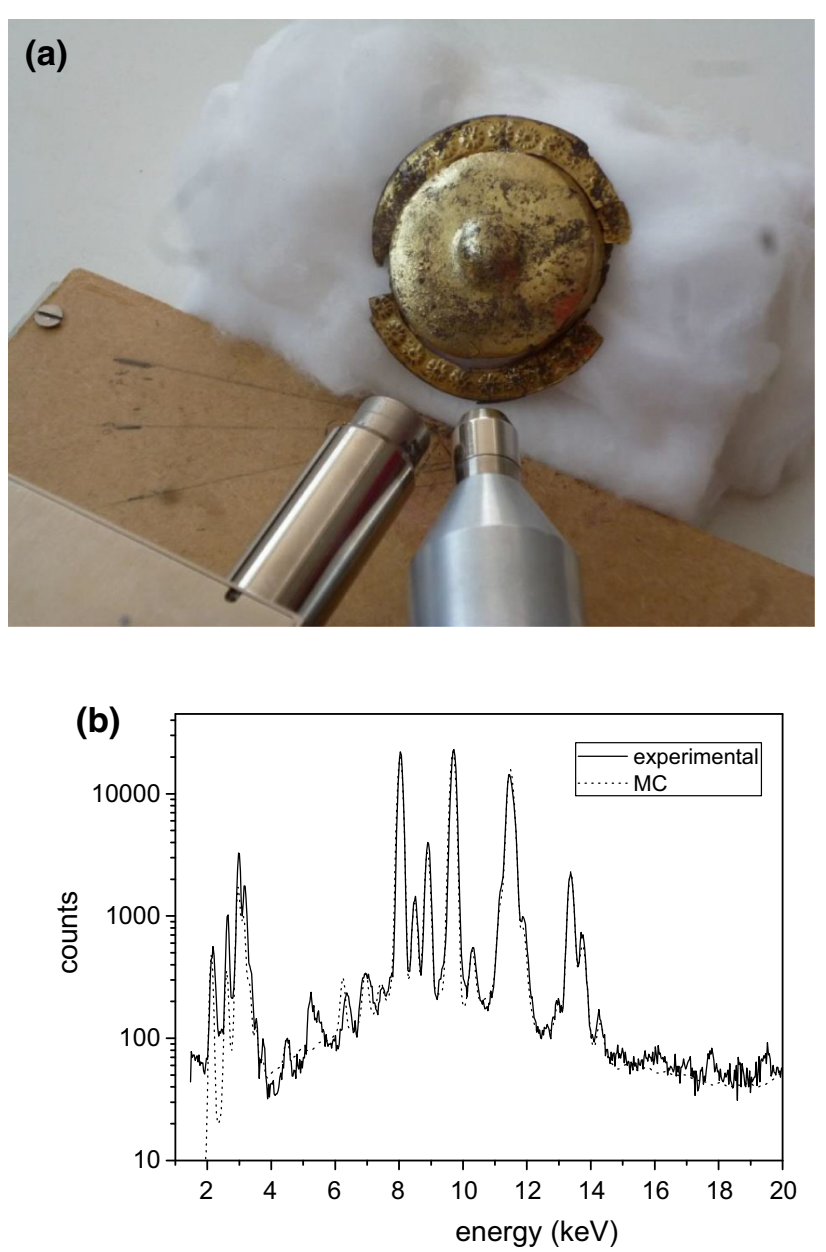

Fig. 6 Gold earring. a Experimental setup; b experimental spectrum and MC simulation 

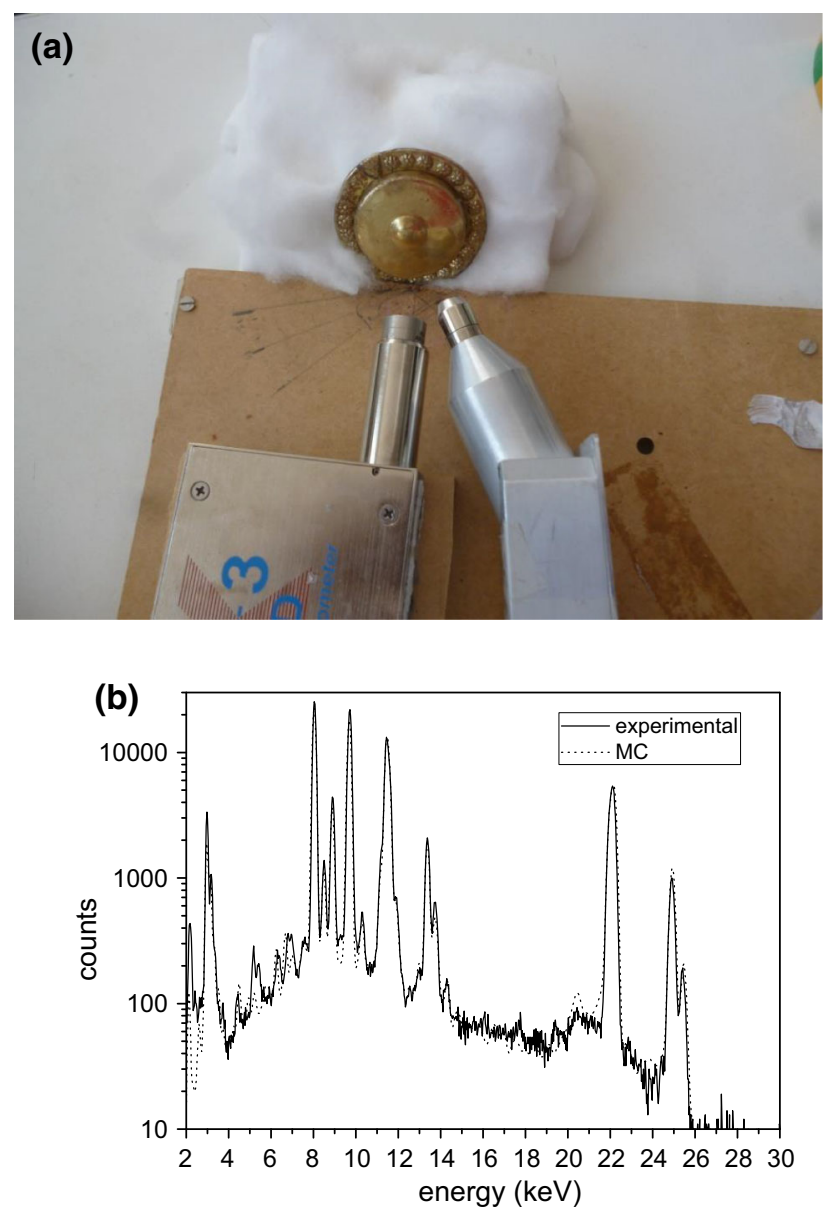

Fig. 7 Gold earring. a Experimental setup; b experimental spectrum and $\mathrm{MC}$ simulation

In Fig. 6a, the first of the earrings samples is shown. In Fig. 6b, the experimental and the Monte Carlo simulation are reported, each superimposed to other.

This earring displays a very complex structure: a first layer, $20 \mu \mathrm{m}$ thick, made up of a protective layer with inclusion of $\mathrm{Ag}, \mathrm{Cu}$ and $\mathrm{Au}$ besides other elements in minor concentrations; a second layer, $2 \mu \mathrm{m}$ thick, formed essentially by $\mathrm{Au}$; and a third layer formed by $\mathrm{Ag}$ and $\mathrm{Au}$. It appears to be an example of depleted alloy, with the silver having been removed from the surface as no gilding has been observed. In this case, a small amount of chlorine and sulfur was also noticed.

In Fig. 7a, b, the picture of another earring, the experimental and MC simulation spectrum are reported. Despite the fact that its appearance is very similar to the previous earring, its conservation state is better than the other one. The object appears to be formed by a $100-\mu \mathrm{m}$-thick Paraloid layer, a 2- $\mu \mathrm{m}$-thick layer $(\mathrm{Cu}(20 \%), \mathrm{Br}(1.0 \%), \mathrm{Au}$ $(79 \%)$ ) and a $\mathrm{Cu}(40.0 \%)$ and $\mathrm{Ag}(60 \%)$ layer, without the presence of gold in appreciable concentrations. Of course in
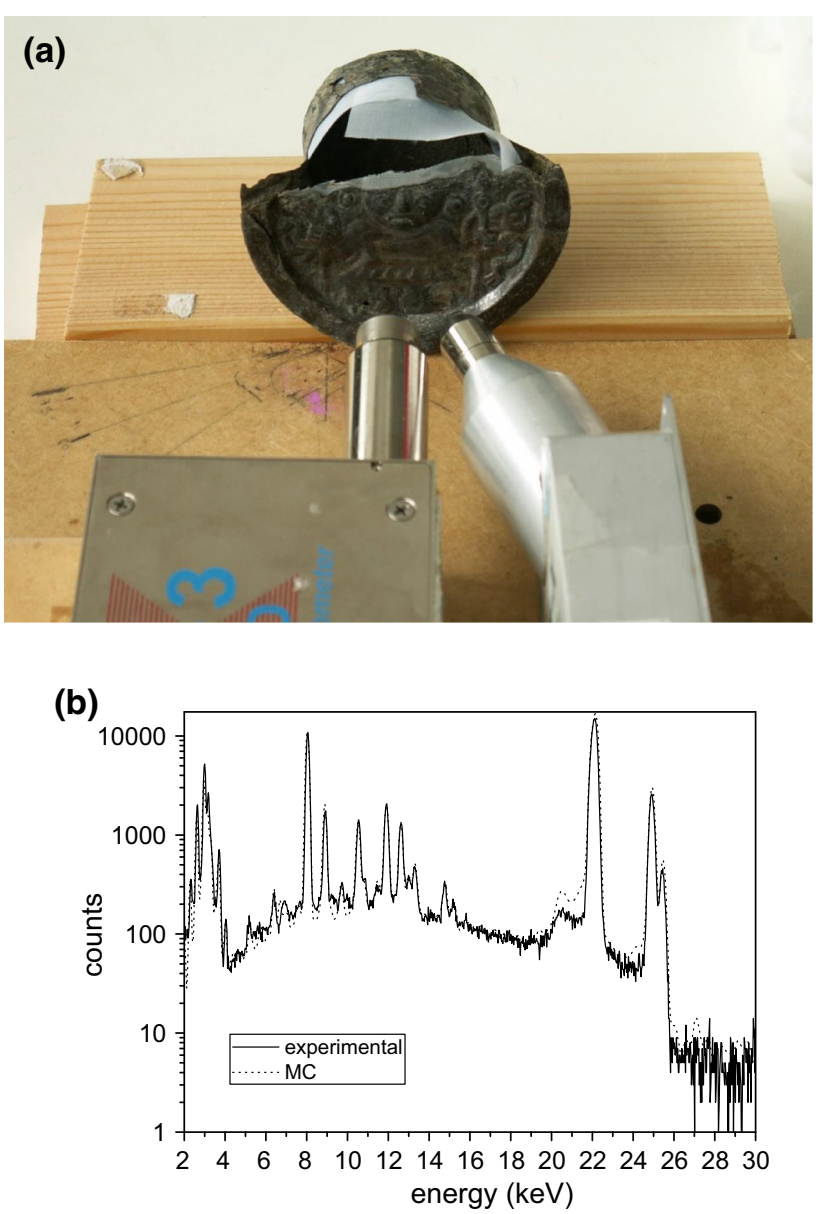

Fig. 8 Silver earring. a Experimental setup; b experimental spectrum and MC simulation

this case too, several other structure/composition sample models have been considered. At a visual examination, the earring structure found in the previous example is similar to the one in Fig. 6a, but the Ag and the Au concentrations in the first layer are considerably lower and, in this case, the protective layer does not show any contamination. Thus, in this case, we have two similar earrings, but in a different state of conservation. Even in this case, a small amount of chlorine and sulfur was also noticed.

In Fig. 8, the last of the earrings studied here is shown together with the experimental and MC simulation. In this case, the object appears to be formed by a $100-\mu \mathrm{m}$-thick chlorine and sulfur-rich surface layer and a second layer essentially formed by Ag. The first layer contains Ag and $\mathrm{Cu}$ both as sulfides and as chlorides, together with $\mathrm{Br}$ (found in many other objects in the burial). The second layer, besides $\mathrm{Ag}(89 \%)$, contains also $\mathrm{Cu}(10 \%)$ and $\mathrm{Ni}$, $\mathrm{Fe}, \mathrm{Bi}, \mathrm{Pb}$ and $\mathrm{Bi}$ in minor concentrations $(<1 \%)$. Thus, this earring can be considered as an almost pure $\mathrm{Ag}$ artifact. 


\section{Conclusions}

In this paper an innovative analytical approach combining XRF measurements with an ultra-fast Monte Carlo simulation has been applied to study selected metal objects from the tomb of Chornancap Priestess in Peru. It allows for a simultaneous description of the composition and the structure of the object examined, and it is of particular importance for this class of samples that are always composed of complex multilayer structures. It is also possible to confirm the archeological hypothesis regarding the technology used, i.e., essentially about Tumbaga or gilding, while in other cases our XRF studies have given a different estimate of composition and structure with respect to the expected one. The tool can be also used before restoration, thus acting as an auxiliary tool for restorers. In the near future, all the samples from the sepulture will be examined as well as some previously examined (with standard XRF approaches) samples from other sites to gain a better understanding of the technology used by the ancient civilization in Peru.

\section{References}

1. R. Cesareo, A. Castellano, G. Buccolieri, S. Quarta, M. Marabelli, P. Santopadre, M. Leole, A. Brunetti, Portable equipment for energy dispersive X-ray fluorescence analysis of Giotto's frescoes in the Chapel of the Scrovegni. Nucl. Instrum. Methods Phys. Res. Sect. B 213, 703-706 (2004)

2. A. Guilherme, M. Manso, S. Pessanha, A. Zegzouti, M. Elaatmani, R. Bendaoud, J. Coroado, J.M.F. dos Santos, M.L. Carvalho, Micro-XRF for characterization of Moroccan glazed ceramics and Portuguese tiles. J Instrum 8, C02055 (2013)

3. R. Cesareo, C. Calza, M. Dos Anjos, R.T. Lopes, A. Bustamante, S.J. Fabian, W. Alva, Z.L.R. Chero, Pre-Columbian alloys from the royal tombs of Sipan; energy dispersive X-ray fluorescence analysis with a portable equipment. Appl. Radiat. Isot. 68, 525-528 (2010)

4. R. Cesareo, A. Bustamante, J. Fabian, C. Calza, M. Dos Anjos, R.T. Lopes, W. Alva, L. Chero, M. Espinoza, R. Gutierrez, R. Rodriguez, M. Seclen, Portable equipment for a non-destructive analysis of pre-Columbian metal artefacts from the Royal Tombs of Sipan by energy-dispersive X-ray fluorescence spectrometry. X-Ray Spectrom. 40, 37-46 (2011)

5. R. Cesareo, M.A. Rizzutto, A. Brunetti, D.V. Rao, Metal location and thickness in a multilayered sheet by measuring $\mathrm{K} \alpha / \mathrm{K} \beta, \mathrm{L} \alpha /$ $\mathrm{L} \beta$ and $\mathrm{L} \alpha / \mathrm{L} \gamma \mathrm{X}$-ray ratios. Nucl. Instrum. Methods Phys. Res. Sect. B 267, 2890-2896 (2009)

6. R. Cesareo, A. Brunetti, S. Ridolfi, Pigment layers and precious metal sheets by energy-dispersive X-ray fluorescence analysis. X-Ray Spectrom. 37, 309-316 (2008)

7. R. Cesareo, A. Brunetti, Metal sheets thickness determined by energy-dispersive X-ray fluorescence analysis. J. X-Ray Sci. Technol. 16, 119-130 (2008)

8. A. Bustamante, R. Cesareo, A. Brunetti, M. Rizzutto, C. Calza, R. Pereira de Freitas, U. Holmsquit, D. Diestra, Analysis of PreColumbian objects from Cupisnique, one of the oldest culture from Perú, using a portable X-ray fluorescence equipment. Appl. Phys. A Mater. Sci. Process. 113, 1065-1067 (2013)

9. R. Cesareo, A. Brunetti, R. D’Oriano, A. Canu, G.M. Demontis, A. Celauro, A Roman bronze statuette with gilded silver mask from Sardinia: an EDXRF study. Appl. Phys. A Mater. Sci. Process. 113, 905-910 (2013)

10. N. Schiavon, A. Celauro, M. Manso, A. Brunetti, F. Susanna, Early Iron Age bronze statuettes in Southern Portugal: combining archaeological data with EDXRF and BSEM + EDS to assess provenance and production technology. Applied Physics Part A 113(4), 865-875 (2013). doi:10.1007/s00339-013-7747-7

11. N. Schiavon, V. Soria, A. M. Arruda, M. Beltrame, J. Mirão, "Losanga" decorated Imitations of Italic Late Republican Black Gloss Tableware from South-Western Iberia: a multi-analytical/ microchemical characterization. Microchem. J. 124, 712-718 (2016)

12. C. Bottaini, J. Mirão, M. Figuereido, A. Candeias, A. Brunetti, N. Schiavon, Energy dispersive X-ray fluorescence Spectroscopy/ Monte Carlo simulation approach for the non-destructive analysis of corrosion patina-bearing alloys in archaeological bronzes: the case of the bowl from the Fareleira 3 site (Vidigueira, South Portugal). Spectrochim. Acta B 103-104, 9-13 (2015)

13. M. Manso, N. Schiavon, I. Queralt, A.M. Arruda, J.M. Sampaio, A. Brunetti, Alloy characterization of a 7th Century BC archaeological bronze vase-overcoming patina constraints using Monte Carlo simulations. Spectrochim. Acta B 107, 93-96 (2015). doi:10.1016/j.sab.2015.03.001

14. G. Marchand, E. Guilminot, S. Lemoine, L. Rossetti, M. Vieau, N. Stephant, Degradation of archaeological horn silver artefacts in burials. Herit. Sci. 2, 5 (2014)

15. R.T. Cesareo, J.T. de Assis, C. Roldán, C. Bustamante, A. Brunetti, N. Schiavon, Multilayered samples reconstructed by measuring $\mathrm{Ka} / \mathrm{K} \beta$ or $\mathrm{La} / \mathrm{L} \beta$ X-ray intensity ratios by EDXRF. Nucl. Instrum. Methods Phys. Res. Sect. B 312, 15-22 (2013)

16. R.P. Gardner, J.M. Doster, Reduction of matrix effects in x-ray fluorescence analysis by the monte carlo, fundamental parameters method. Adv. X-Ray Anal. 22, 343-356 (1979)

17. R.P. Gardner, J.M. Doster, The complete spectral response for EDXRF systems-calculation by Monte Carlo and Analysis applications. 1 Homogeneous samples. X-ray Spectro. 11, 173-180 (1982)

18. R.P. Gardner, J.M. Doster, The complete spectral response for EDXRF systems-calculation by Monte Carlo and Analysis applications. 1 Heterogeneous samples. X-Ray Spectrom. 11, 181-186 (1982)

19. J.E. Fernandez, Monte Carlo computer simulation of the XRF intensity dependence on the propagation plane inclination. Comput. Phys. Commun. 54, 211-220 (1989)

20. U. Bottigli, A. Brunetti, B. Golosio, P. Oliva, S. Stumbo, L. Vincze, P. Randaccio, P. Bleuet, A. Simionovici, A. Somogyi, Voxel-based Monte Carlo simulation of X-ray imaging and spectroscopy experiments. Spectrochim. Acta B 59, 1747-1754 (2004)

21. B. Golosio, A. Brunetti, G.L. Masala, P. Oliva, T. Schoonjans, Monte Carlo simulation of X-ray imaging and spectroscopy experiments using quadric geometry and variance reduction techniques. Comput. Phys. Commun. 185, 1044-1052 (2014)

22. L. Vincze, K. Janssen, F. Adams, A general Monte Carlo simulation of energy-dispersive X-ray fluorescence spectrometers-I: unpolarized radiation, homogeneous samples. X-Ray Spectrom. 48, 553-573 (1993)

23. T. Schoonjans, L. Vincze, V.A. Solé, M. Sanchez del Rio, P. Brondeel, G. Silversmit, K. Appel, C. Ferrero, A general Monte Carlo simulation of energy-dispersive X-ray fluorescence spectrometers-Part 5. Polarized radiation, stratified samples, cascade effects, M-lines. Spectrochim. Acta, B 70, 10-23 (2012) 
24. A. Brunetti, M. Sanchez del Rio, B. Golosio, A. Simionovici, A. Somogyi, A library for X-ray-matter interaction cross sections for X-ray fluorescence applications. Spectrochim. Acta B 59, 1725-1731 (2004)

25. T. Schoonjans, A. Brunetti, B. Golosio, M. Sanchez del Rio, V.A. Solé, C. Ferrero, L. Vincze, The xraylib library for X-ray-matter interactions. Recent developments. Spectrochim. Acta Part B 66, 776-784 (2011)

26. R.F.M. Hedges, On the occurrence of bromine in corroded silver. Stud. Conserv. 21, 44-46 (1976) 